\title{
EL SECTOR PRIMARIO Y EL ESTANCAMIENTO ECONÓMICO EN MÉXICO
}

\author{
Moritz Cruz y Mayrén Polanco*
}

Fecha de recepción: 10 de febrero de 2014. Fecha de aceptación: 11 de abril de 2014.

\begin{abstract}
RESUMEN
Como bien se sabe, un sector primario capaz de generar un excedente de producción permanentemente es indispensable para el desarrollo económico sostenido. El estancamiento mexicano iniciado durante la década de los ochenta, se ha caracterizado, entre otros elementos, por el pobre desempeńo del sector primario. Esto permite plantear la interrogante sobre el papel que tal sector ha realizado en dicho estancamiento. En este trabajo, con el apoyo de técnicas econométricas, se intenta responder a dicha pregunta usando datos para el periodo 1970-2012. Los resultados sugieren una contribución negativa del sector primario al crecimiento económico; asimismo, su desempeño ha acentuado las restricciones al crecimiento impuestas por el modelo de crecimiento vigente.
\end{abstract}

Palabras clave: México, sector primario, crecimiento económico, desarrollo económico, tasa de crecimiento.

Clasificación JEL: C01, E22, F43, N56, O13, Q18.

\section{The Primary Sector and Economic Stagnation in Mexico}

\begin{abstract}
As is widely known, a primary sector consistently able to generate a production surplus is indispensable for sustained economic growth. The stagnation that began in Mexico in the 1980s has been characterized by the poor performance of the primary sector, among other elements. It is therefore interesting to explore the role this sector has played in stagnation. This work examines this topic using data for the time period 1970-2012 and some econometric techniques. The results suggest that the primary sector has made a negative contribution to economic growth, while primary sector performance has aggravated limitations on growth imposed by the current growth model.
\end{abstract}

Key Words: Mexico, primary sector, economic growth, economic development, growth rate.

\footnotetext{
* Instituto de Investigaciones Económicas-unam, aleph398@gmail.com y Universidad de Colima, México, mayrenpg@gmail.com, respectivamente.
} 


\section{LE SECTEUR PRIMAIRE ET LA STAGNATION ÉCONOMIQUE AU MEXIQUE Résumé}

Comme il est de notoriété, un secteur primaire capable de générer un excédent de production en permanence est indispensable pour le développement économique durable. Les faibles performances du secteur primaire a été une des caractéristiques de la période de stagnation qui a commencé au Mexique dans les années 80 . Ceci permet de poser la question du rôle qu'a joué ce secteur dans cette stagnation. Ce travail essaie de répondre à cette question à l'aide de techniques économétriques en se fondant sur des données pour la période 1970-2012. Les résultats suggèrent une contribution négative du secteur primaire à la croissance économique ; ainsi donc, ses faibles performances ont accentué les restrictions à la croissance imposées par le modèle de croissance en vigueur.

Mots clés: Mexique, secteur primaire, croissance économique, développement éconiomique, taux de croissance.

\section{O SETOR PRMÁRIO E A ESTAGNAÇÃO ECONÔMICA NO MÉXICO Resumo}

Como é bem sabido, um setor primário capaz de gerar excedente de produção permanente é indispensável para o contínuo desenvolvimento econômico. A estagnação mexicana iniciada durante a década de oitenta se caracterizou, entre outros elementos, pelo pobre desempenho do setor primário. Isso permite questionar sobre o papel que tal setor teve na mencionada estagnação. Neste trabalho, com apoio de técnicas econométricas, se trata de responder a essa pergunta usando dados para o período 1970-2012. Os resultados sugerem uma contribuição negativa do setor primário ao crescimento econômico; bem como a inferência que seu desempenho acentuou as restriçôes ao crescimento impostas pelo modelo de crescimento vigente. Palavras-chave: México; setor primário; crescimento económico; desenvolvimento económico; taxa de crescimento.

\section{墨西哥的初级产品行业与经济停滞}

摘要

众所周知, 初级产品行业能够连续不断带来生产剩余, 这对经济可持续增 长必不可少。墨西哥经济停滞始于20世纪80年代, 其特点是初级产品行业 与其他产业相比, 发展表现糟糕。因此, 探讨初级产品行业在, 墨西哥经济 停滞中的角色是一个有兴趣的研究课题。本文使用了1970 2012年数据和 一些计量经济学方法研究了这一问题。研究结论认为, 初级产品行业对经 济增长的贡献是负值, 而初级产品行业的发展表现在当前的增长模式下更 加抑制了对增长的贡献。

关键词：墨西哥 初级产品行业经济增长经济发展 增长率 


\section{INTRODUCCIÓN}

La contribución positiva que el sector primario hace consistentemente al crecimiento económico es uno de los argumentos sobre el que hay un claro consenso en la literatura sobre el crecimiento y desarrollo económico. ${ }^{1}$ Se reconoce, en otras palabras, que el sector primario contribuye siempre de manera positiva al crecimiento económico. Entre los trabajos recientes que arrojan evidencia empírica soportando dicho argumento destacan los de Tiffin e Irz (2006), Yao (2000), Gollin et al. (2002), y Henley (2012).

La contribución que hace el sector primario ocurre mediante diferentes vías -en general, identificadas como factorial, de divisas, de mercado y de producción-, cuyo peso evoluciona con el transcurso del desarrollo económico. Así, durante las etapas iniciales, la contribución del sector primario no sólo es elevada sino crucial para el éxito económico. De hecho, en estas etapas se sostiene que el sector primario es la base para que el sector industrial se fortalezca y expanda, permitiéndole ser eventualmente el nuevo motor del crecimiento económico (véase Kaldor, 1966, 1989). Más tarde, al alcanzarse la fase de industrialización madura, se espera que el sector primario deje de contribuir en la misma medida al éxito económico; no obstante, en ésta, y posteriores etapas, continuará haciendo una contribución tanto positiva como estratégica, principalmente por la vía del producto y de las divisas. Más aún, a partir de cierta etapa de industrialización, es posible esperar que el sector primario se convierta en receptor de recursos del resto de la economía, vía subsidios y otros incentivos fiscales, con el objeto de mantener los excedentes productivos que seguirán contribuyendo al crecimiento económico. La continua expansión del sector primario es, en suma, crucial para alcanzar y sostener la dinámica del crecimiento.

A sabiendas de lo anterior, muchas de las naciones que adoptaron el llamado modelo de crecimiento liderado por las exportaciones a partir de la década de 1980 hicieron hincapié en el sesgo anti-rural del mismo (Bezemer y Headey, 2008); como resultado, su crecimiento económico se mantiene estancado. ${ }^{2}$ Es decir, yendo contra toda lógica económica, dichas economías abandonaron a su suerte al sector primario, implicando la reducción significativa de la política agrícola en un contexto de rápida apertura a la competencia internacional y, en muchos casos, de modificaciones a la legislación agraria para permitir, entre otras, la concentración de las tierras en manos de pocos. Estas medidas tenían por objeto incrementos en la productividad agrícola motivados por una mayor rentabilidad.

Un caso digno de análisis es el de la economía mexicana, que desde la adopción del modelo señalado, después de la crisis de la deuda de 1982, se 
ha caracterizado por tener, paralelo al estancamiento del sector primario, un crecimiento económico pobre. ${ }^{3}$ ¿Es posible, entonces, que dicho desempeño agregado esté fincado también en el estancamiento del sector primario? El objetivo de este trabajo es investigar al respecto, partiendo de la hipótesis de que el sector primario ha dejado de contribuir al crecimiento económico. Para comprobar tal hipótesis, se procede de dos maneras. Primero, por medio de un análisis meramente descriptivo, se intenta conocer cuál ha sido la contribución del sector primario al desarrollo económico. En segundo lugar, siguiendo el trabajo de Yao (2000), se identifica el tipo de relación que el sector primario mantiene en el largo plazo con el resto de los sectores productivos con el fin de validar el análisis descriptivo previo. Se considera que este ejercicio es relevante pues, de comprobarse la hipótesis, se fortalece la necesidad de incluir el impulso y apoyo irrestricto al sector primario en una estrategia de crecimiento como condición sine qua non para su éxito.

El trabajo contiene, además de esta introducción, dos secciones adicionales. La primera, describe brevemente los mecanismos por los cuales el sector productivo promueve el crecimiento económico. La segunda, presenta evidencia descriptiva sobre el papel que el sector primario ha tenido en la economía mexicana desde el último tercio del siglo pasado. Esta sección también presenta y discute los resultados de las estimaciones econométricas que permiten identificar la contribución del sector primario al crecimiento económico durante el periodo 1970-2012. La última sección son conclusiones.

\section{LA CONTRIBUCIÓN DEL SECTOR PRIMARIO AL CRECIMIENTO ECONÓMICO}

El objetivo de esta sección es recordar brevemente cuáles son los mecanismos por los cuales el sector primario contribuye al crecimiento económico. Antes de describirlos, es importante insistir en que conforme la economía transita a etapas más avanzadas de industrialización es de esperarse que dicha contribución evolucione en forma decreciente. Lo relevante a destacar en este contexto es que mientras la contribución al crecimiento del sector primario durante etapas iniciales de desarrollo suele ocurrir de manera "natural" debido a la importancia del sector en la economía (tanto en términos de producto como de empleo), en etapas posteriores, cuando se reduce su tamaño, dicha contribución es positiva sólo si dicho sector mantiene el apoyo del resto de la economía; es decir, sólo si se mantienen los mecanismos -léase una política agraria activa- para continuar generando un excedente. 
Aunque diversos, los canales por los cuales una economía contribuye al crecimiento económico pueden, en general, agruparse en cuatro, a saber el factorial, el de las divisas, el del mercado y el de la producción (véase, por ejemplo, Adelman, 1984; Yao, 2000; Thirlwall, 2003; Moreno-Brid y Ros, 2009; Calva, 2012). ${ }^{4}$ La contribución factorial hace referencia a todos aquellos factores productivos que el sector primario libera o produce para el resto de los sectores productivos. Por ejemplo, al ser abundante en mano de obra, es factible que el sector primario libere o transfiera fuerza de trabajo a bajo costo, sin que su productividad necesariamente caiga (à la Lewis, 1954). Al mismo tiempo, produce la materia prima que, en las etapas iniciales de industrialización, son básicas para las nacientes empresas. Por otra parte, el sector primario es fuente de recursos financieros (particularmente por medio del ahorro forzoso) para solventar los diversos proyectos de inversión. En este contexto, no debe olvidarse que mediante las variaciones de los términos de intercambio entre bienes primarios e industriales el sector primario también contribuye en el financiamiento de la inversión en otros sectores.

El sector primario, por otra parte, contribuye al creciemiento económico por medio de las divisas que aporta para financiar los requerimientos de importaciones del conjunto de la economía, particularmente de bienes de capital. En este contexto, no debe olvidarse la contribución que el sector primario hace al crecimiento por la vía de la balanza de pagos. El mecanismo es muy simple: el saldo comercial positivo del sector primario, contribuye al saldo (positivo o negativo) de la balanza comercial total, aliviando así cualquier restricción al crecimiento por la vía de la balanza de pagos. Esta contribución no es de ninguna manera trivial ya que la restricción al crecimiento de la balanza de pagos ha sido uno de los problemas más acuciantes de las economías en desarrollo durante las últimas décadas (McCombie y Thirlwall, 2004).

Otra contribución ocurre por la vía del mercado que representa para la producción industrial, especialmente en las etapas iniciales de la industrialización. Por un lado, los trabajadores del sector primario constituyen el mercado natural, que inicialmente consume la producción del sector industrial. Por el otro, la creciente productividad del sector primario suele anclarse en los insumos mejorados que el sector industrial provee, generándose así fuertes encadenamientos productivos que inciden en la dinámica de crecimiento del sector industrial. El mercado primario, en suma, constituye la base para el despegue industrial. Gracias a éste, el sector industrial, en general, y el manufacturero, en particular, se convertirán en el motor del crecimiento económico (Kaldor, 1966). ${ }^{5}$ Es decir, sin la contribución inicial del sector primario, es virtualmente imposible transitar hacia etapas posteriores de industrialización. 
Finalmente, el sector primario provee de la comida necesaria para una población que, conforme se emplea y crece su ingreso, demandará cantidades y variedades mayores de alimento (Huang y Bouis, 2001). La capacidad de una economía para satisfacer la demanda de alimentos es sin duda relevante pues implica, entre otros aspectos fundamentales, evitar, tanto la dependencia alimentaria como las potenciales espirales inflacionarias con sus consabidas consecuencias negativas en otras variables económicas (como el tipo de cambio real) y en la restricción interna al crecimiento (Cruz et al., 2011).

Así, en suma, la contribución del sector primario al crecimiento económico, particularmente durante las etapas iniciales de industrialización, es ingente y fundamental para el éxito económico. Desde luego, y como señalamos, conforme la industrialización progresa, su contribución disminuye en tamaño, pero no en importancia. Así, por ejemplo, un sector primario en continua expansión (al menos en términos de producción) puede mantener satisfecha la demanda interna por alimentos y, al mismo, tiempo exportar el exceso de la producción. Esto se traduce en una contribución al crecimiento por la vía de las divisas y de la producción.

Ahora bien, es importante señalar que todos los beneficios descritos se derivan del supuesto de que la productividad del sector primario es en promedio creciente (es decir, que se ha transitado de la agricultura tradicional a una altamente mecanizada y que utiliza insumos mejorados), traduciéndose en un excedente de producción (mismo que se exporta o bien gracias al cual es posible liberar mano de obra). Como lo demuestra la evidencia de cualquier país exitoso, dicho excedente sólo es asequible permanentemente cuando el gobierno brinda su apoyo al sector por medio de los diferentes mecanismos que en conjunto se conocen como política agraria. Ésta involucra desde los subsidios hasta los precios de garantía, pasando por el apoyo en la investigación, el acceso al crédito y a los seguros, el desarrollo de infraestructura, etc. En este sentido, el auge constante del sector primario -mismo que reside esencialmente en el suministro de mejores insumos y en la aplicación del nuevo conocimiento y de tecnología moderna (Thirlwall, 2003: 204)-, y su consecuente contribución al crecimiento, descansa sobre la base de una política agraria activa.

Es de esperarse, dado todo lo anterior, que el desmantelamiento de la política agraria desemboque en el estancamiento económico y/o el reforzamiento de las restricciones al crecimiento económico. Lo anterior, independientemente del modelo económico que se haya adoptado (sustitución de importaciones o crecimiento liderado por las exportaciones). 


\section{ESTANCAMIENTO ECONÓMICO Y SECTOR PRIMARIO: EVIDENCIA PARA EL PERIODO 1970-2012}

A principios de la década de 1980, con la deuda de la crisis emergiendo, la economía mexicana dejo atrás cuatro décadas de éxito económico (1940-1980), para iniciar, con la adopción de un modelo de crecimiento liderado por las exportaciones, una etapa marcada por la inestabilidad y por el estancamiento económico, misma que se ha prolongado ya por más de tres décadas.

Es importante notar que durante el periodo de éxito económico, con un modelo de sustitución de importaciones en marcha, el sector primario registró una época de bonanza. De acuerdo con Calva (2012: 69) “[...] el PIв agrícola [...] creció a una tasa media de 5.5\% anual entre el trienio 1939-1941 y el de 1964-1966; y el PIB agropecuario, forestal y pesquero creció a una tasa media de 4.6\% anual durante el mismo lapso". De hecho, durante el periodo 19401958 el pIB primario creció en promedio anual 7\%, prácticamente a la misma tasa que el conjunto de la economía (que en estos años era meramente agraria e impulsada por dicho sector); mientras que de 1959 a 1981 el PIB agropecuario creció por debajo del agregado ${ }^{6}$ (Gómez-Oliver, 1996), mismo que era impulsado ya por la dinámica industrial (Moreno-Brid y Ros, 2009).

Gracias entonces a la dinámica del sector primario, el conjunto de la economía pudo avanzar sostenidamente en su proceso de industrialización. Es decir, durante el periodo de éxito económico, el sector primario contribuyó en forma importante al crecimiento económico por las vías destacadas en la sección previa. Por ejemplo, la contribución del sector primario por la vía factorial al conjunto de la economía durante el periodo 1940-1960, se tradujo en un empuje de la inversión de 2 a 3\% del total de la inversión fija durante ese periodo (Eckstein, 1968; Moreno-Brid y Ros, 2009). Asimismo, el crecimiento agrícola significó la fuente primordial de divisas para cubrir las importaciones de capital, satisfizo la creciente demanda interna por alimentos, proveyó las materias primas que demandaba la naciente industria y liberó mano de obra abundante para el resto de los sectores productivos (Calva, 2012: 84-85).

Desde luego, lo anterior no fue providencial sino el resultado de una política agrícola que incluyó los típicos instrumentos de fomento económico sectorial, es decir, la construcción pública de infraestructura, la investigación y la transferencia de tecnología, el crédito preferencial y los seguros agropecuarios apoyados con recursos públicos, los subsidios a insumos agrícolas y un sistema de precios garantía (Calva, 2012: 68-69; y 1988). Durante el periodo 19341950 , por ejemplo, la inversión pública canalizada a la agricultura aumentó en términos reales $7 \%$ promedio anual, y entre 1957 y 1981 creció a una tasa 
anual superior a 10\%. Más aún, los subsidios que el sector recibió crecieron a una tasa anual de $12.5 \%$ entre 1970 y 1981, llegando a representar $20 \%$ del PIB agrario y casi $2 \%$ del agregado (Gómez-Oliver, 1996).

Contrariamente, y por diversas causas, ${ }^{7}$ la instauración del modelo de crecimiento liderado por las exportaciones a principios de la década de los ochenta se caracterizó por su sesgo anti-rural, implicando la profundización ${ }^{8}$ en el desmantelamiento de la política agrícola. Como resultado, el producto agrícola aceleró la caída que había iniciado desde mediados de la década de los sesenta debida, principalmente, al descenso de la inversión en el sector como resultado de la disminución de su rentabilidad (Calva, 2012; Gómez-Oliver, 1996). Al respecto, de 1970 a 2011, el PIB primario creció apenas a una tasa cercana de $2 \%$ promedio anual, muy por debajo de los estándares registrados (mencionados arriba) en la época de éxito económico. De hecho, como se aprecia en la gráfica 1 , la tendencia del crecimiento del sector primario es hacia la baja desde inicios de la década de los sesenta (con una ligera recuperación en esos años) y hasta finales de la de los ochenta; posteriormente, es posible observar un repunte que se estanca rápidamente y que perdura hasta el final de la década de los noventa para, desde entonces, iniciar un nuevo declive y una moderada ulterior recuperación.

Gráfica 1. Tasa de crecimiento del sector primario y su tendencia, 1971-2011

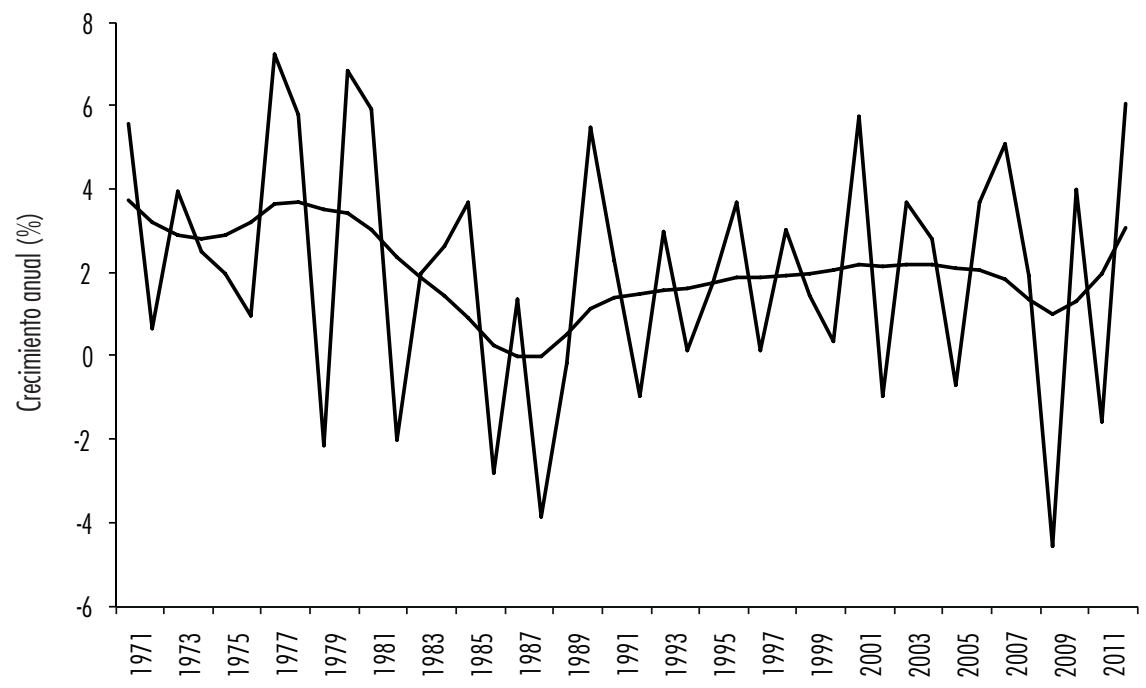

Fuente: Elaboración propia con la base de datos de las Naciones Unidas (disponible en: unstats.un.org). 
Con relación al desmantelamiento de la política agrícola, el cuadro 1 ilustra claramente la medida en que uno de los instrumentos más relevantes de dicha política, el crédito oficial, fue abruptamente reducido. Si observamos la columna tres, podemos notar que desde 1980 el crédito oficial al sector muestra en general una tendencia a la baja, misma que en ningún momento recupera los niveles iniciales del periodo de análisis. La información del cuadro 1 también es indicativa de que la principal fuente de crédito para el sector empezó a recaer en la banca comercial, no obstante sólo durante el periodo 1990 a 1994 es posible identificar un aumento sostenido de dicho crédito. ${ }^{9}$

Otro indicador relevante del desmantelamiento de la política agraria se refleja en la caída en los montos de uno de los instrumentos pilares de la política agraria: los subsidios. En efecto, a partir de la crisis de 1982 tanto los subsidios como los gastos en fomento agrícola se redujeron aceleradamente a menos de la mitad, siguiendo una tendencia marcadamente decreciente. Para 1987 el total del subsidio a la agricultura era inferior a medio punto del PIB total. En este contexto, el gasto público canalizado a la agricultura disminuyó rápidamente: de $12 \%$ en 1980 a 9.6\% en 1983 y a menos de 6\% en 1989 (GómezOliver, 1996) (véase el cuadro 2).

Cuadro 1. Crédito otorgado al sector agropecuario, 1980-2012

(saldos al final del periodo en millones de pesos de 2010)*

\begin{tabular}{lccc}
\hline Año & Crédito total & Banca comercial & Banca de desarrollo \\
\hline 1980 & 208587 & n.d & n.d \\
1981 & 200667 & n.d & n.d \\
1982 & 129552 & n.d & n.d \\
1983 & 109092 & n.d & n.d \\
1984 & 125353 & 8665 & 6.9 \\
1985 & 124205 & 9154 & 7.4 \\
1986 & 86630 & 7107 & 8.2 \\
1987 & 67869 & 4182 & 6.2 \\
1988 & 99766 & 4790 & 4.8 \\
1989 & 137060 & 14164 & 10.3 \\
& & & (Continúa)
\end{tabular}


Moritz Cruz y Mayrén Polanco

(Continuación cuadro 1)

\begin{tabular}{|c|c|c|c|}
\hline Año & Crédito total & Banca comercial & Banca de desarrollo \\
\hline 1990 & 166714 & 21712 & 13.0 \\
\hline 1991 & 163046 & 15499 & 9.5 \\
\hline 1992 & 195732 & 19681 & 10.1 \\
\hline 1993 & 222351 & 37560 & 16.9 \\
\hline 1994 & 260421 & 41077 & 15.8 \\
\hline 1995 & 185168 & 56787 & 30.7 \\
\hline 1996 & 171519 & 60046 & 35.0 \\
\hline 1997 & 159144 & 73660 & 46.3 \\
\hline 1998 & 116552 & 76061 & 65.3 \\
\hline 1999 & 93971 & 57584 & 61.3 \\
\hline 2000 & 76906 & 44433 & 57.8 \\
\hline 2001 & 65917 & 37566 & 57.0 \\
\hline 2002 & 50735 & 27674 & 54.5 \\
\hline 2003 & 45056 & 28422 & 63.1 \\
\hline 2004 & 26333 & 7702 & 29.2 \\
\hline 2005 & 27069 & 2684 & 9.9 \\
\hline 2006 & 26587 & 1456 & 5.5 \\
\hline 2007 & 32290 & 892 & 2.8 \\
\hline 2008 & 36728 & 975 & 2.7 \\
\hline 2009 & 31304 & 1532 & 4.9 \\
\hline 2010 & 34020 & 1617 & 4.8 \\
\hline 2011 & 39412 & 1596 & 4.0 \\
\hline 2012 & 47323 & 2146 & 4.5 \\
\hline
\end{tabular}

*Deflactado con el índice nacional de precios del consumidor (2010=100); n.d.: no disponible.

Fuente: Elaboración propia con base en Banco de México, Indicadores económicos y Calva (2007: 32-33). 
Cuadro 2. Inversión y gasto público en el sector agropecuario y forestal, 1980-2011 (porcentaje del PIB)

\begin{tabular}{|c|c|c|}
\hline Año & Inversión & Gasto \\
\hline 1980 & 2.63 & 1.52 \\
\hline 1981 & 2.63 & 1.17 \\
\hline 1982 & 2.14 & 1.03 \\
\hline 1983 & 1.92 & 0.71 \\
\hline 1984 & 1.73 & 0.68 \\
\hline 1985 & 1.52 & 0.58 \\
\hline 1986 & 1.50 & 0.48 \\
\hline 1987 & 1.09 & 0.39 \\
\hline 1988 & 0.86 & 0.25 \\
\hline 1989 & 0.80 & 0.28 \\
\hline 1990 & 1.58 & 0.27 \\
\hline 1991 & 1.02 & 0.28 \\
\hline 1992 & 1.16 & 0.25 \\
\hline 1993 & 1.29 & 0.18 \\
\hline 1994 & 1.40 & 0.19 \\
\hline 1995 & 1.01 & 0.13 \\
\hline 1996 & 0.96 & 0.16 \\
\hline 1985 & 0.80 & 0.14 \\
\hline 1986 & 0.70 & 0.08 \\
\hline 1987 & 0.52 & 0.06 \\
\hline 1988 & 0.49 & 0.05 \\
\hline 1989 & 0.53 & 0.05 \\
\hline 1990 & 1.22 & 0.05 \\
\hline 1991 & 0.54 & 0.05 \\
\hline
\end{tabular}


Moritz Cruz y Mayrén Polanco

(Continuación cuadro 2)

\begin{tabular}{|c|c|c|}
\hline Año & Inversión & Gasto \\
\hline 1992 & 0.51 & 0.05 \\
\hline 1993 & 0.53 & 0.06 \\
\hline 1994 & 0.49 & 0.05 \\
\hline 1995 & 0.56 & 0.10 \\
\hline 1996 & 0.59 & 0.08 \\
\hline 1997 & 0.63 & 0.08 \\
\hline 1998 & 0.60 & 0.08 \\
\hline 1999 & 0.58 & 0.09 \\
\hline 2000 & 2.63 & 1.52 \\
\hline 2001 & 2.63 & 1.17 \\
\hline 2002 & 2.14 & 1.03 \\
\hline 2003 & 1.92 & 0.71 \\
\hline 2004 & 1.73 & 0.68 \\
\hline 2005 & 1.52 & 0.58 \\
\hline 2006 & 1.50 & 0.48 \\
\hline 2007 & 1.09 & 0.39 \\
\hline 2008 & 0.86 & 0.25 \\
\hline 2009 & 0.80 & 0.28 \\
\hline 2010 & 1.58 & 0.27 \\
\hline 2011 & 1.02 & 0.28 \\
\hline
\end{tabular}

* En 2002 se incluyen 48878 millones de pesos correspondientes a la liquidación de Banrural y a la creación de Financiera Rural.

Fuente: Elaboración propia con base en el INEGI, El ingreso y el gasto público en México, 2005 y 2012; Felipe Calderón , Sexto Informe de Gobierno 2012; e INEGI, Sistema de Cuentas Nacionales. (Los datos de 1980 a 1989 fueron tomados de Calva, 2007: 30 y 31.) 
La puntilla en la desaparición de la pohlítica agraria, y en el consecuente estancamiento del sector primario, fue dada por la unilateral y rápida apertura comercial de que fue objeto la economía mexicana desde mediados de la década de los ochenta, coronada con la firma y entrada en vigor del Tratado de Libre Comercio con Estados Unidos y Canadá a principios de los noventa. En este contexto los precios de garantía o soporte fueron suprimidos, implicando una pérdida tanto en la rentabilidad del sector como en el poder adquisitivo de los productores del campo (Calva, 2012).

Con estos antecedentes, no sería sorprendente observar que la contribución al crecimiento del sector primario dejara de ser positiva, y que las restricciones al crecimiento que el modelo de exportaciones ha ido imponiendo se acentuaran. Un ejemplo al respecto se observa en la evolución del balance comercial total y del sector primario (véase la gráfica 2). En ésta, es evidente que el balance comercial agrario ha sido, desde mediados de la década de los setenta, esencialmente deficitario. Las implicaciones económicas de esta situación son sin duda negativas, y vale la pena resaltarlas. Por un lado, un nulo aporte de divisas para cubrir los requerimientos de importaciones, lo cual ha conllevado al mayor endeudamiento externo o bien a la disminución de las reservas internacionales; ambas opciones, como la evidencia empírica lo señala, son inviables en el largo plazo. Más aún, las recientes crisis económicas de México han estado vinculadas a los insostenibles déficit externos.

Por otra parte, pero relacionado con el punto previo, está el hecho de que el déficit comercial agrario ha contribuido al de la cuenta corriente total, con lo que la restricción al crecimiento por la vía del sector externo no ha podido ser aliviada. En esta tesitura, finalmente, en un estudio reciente Cruz et al. (2011) señalan que la dependencia alimentaria de la economía mexicana es factible activar de la restricción doméstica al crecimiento al ejercer presión al alza en la inflación de origen estructural. Dicha presión inflacionaria puede ser de carácter autónomo, originada en el crecimiento de los precios internacionales de alimentos, o bien de carácter endógeno, debido al caso de un crecimiento económico muy dinámico. Independientemente de su origen, apuntan los autores, la inflación estructural puede, por un lado, incentivar a las autoridades monetarias a frenar el crecimiento económico o, por el otro, afectar negativamente el tipo de cambio real al apreciarlo, resultado en una pérdida de competitividad comercial, y agravando el déficit externo. En suma, la ausencia de un excedente del sector primario ha acentuado, por diversas vías, la activación de mecanismos adversos para el crecimiento de la economía mexicana y ha dejado, al mismo tiempo, de contribuir positivamente al crecimiento económico. 
Gráfica 2. Balanza comercial total y agrícola, 1970-2011 (millones de dólares)

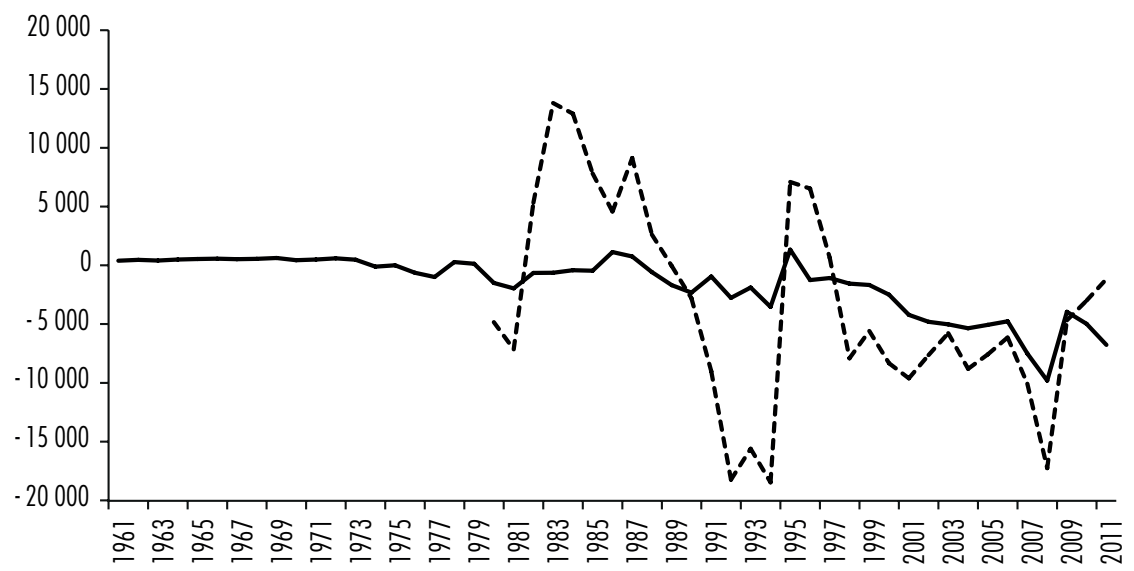

Agrícola ----- Total

Fuente: Estadísticas de la CEPAL (disponible en: estadistical.cepal.org).

Hemos destacado que un mecanismo por el cual el sector primario contribuye al desarrollo es por la vía de la demanda por bienes industriales. Esto es particularmente cierto cuando los ingresos de los trabajadores del sector primario crecen y cuando la participación del empleo del sector en el total es elevada. En este sentido, no es sorprendente que el sector agrario haya dejado de ser una fuente importante de demanda como consecuencia del abrupto freno en el dinamismo del crecimiento económico agregado que se registró a partir de 1982 (Gómez-Oliver, 1996). Es decir, la pérdida de empleo y de poder adquisitivo perpetuadas a partir de 1982 generó la contracción, tanto de la demanda por productos industriales (lo cual a su vez ha afectado negativamente la demanda por materias primas primarias), como la de alimentos (esto es un fenómeno inesperado debido a la baja elasticidad del ingreso de los productos primarios). No debemos olvidar que el sector primario concentra aún a un elevado número de trabajadores -desde inicios de la década de 1980 la participación del empleo del sector primario en el empleo total ha disminuido lentamente, de tal suerte que para 2012 dicha participación se mantenía en alrededor de 14\%-, por lo que la disminución del ingreso en el sector sin duda ha afectado la demanda total. La contracción de la demanda desde mediados de los ochenta ha contribuido en la caída vertiginosa de la participación del sector primario en el PIB total, pasando de $10.6 \%$ del PIB en 1986 a fluctuar alrededor de $3.5 \%$ de éste desde finales de los noventa. Por lo anterior, en términos contables, la contri- 
bución al crecimiento del sector primario es, en general marginal, la misma que excepcionalmente ha alcanzado dos décimas de punto porcentual a partir de la década de los ochenta (véase el cuadro 3).

A la luz de esta evidencia, es claro que el sector primario en México no sólo dejó de contribuir al desarrollo económico sino también ha agudizado las restricciones al crecimiento que el modelo exportador a impuesto. En lo que resta de esta sección, se intenta ilustrar cómo el sector primario está relacionado con el resto de la economía, en particular con el sector industrial; también poder identificar en qué medida está contribuyendo al estancamiento económico mexicano.

Cuadro 3. Contribución de los sectores productivos al producto total

\begin{tabular}{lcccc}
\hline Año & Total & Agricultura & Industria & Servicios \\
\hline 1980 & 8.7 & 0.35 & 2.77 & 5.53 \\
1981 & 8.6 & 0.30 & 3.06 & 5.21 \\
1982 & -0.7 & -0.09 & -0.90 & 0.31 \\
1983 & -4.0 & 0.09 & -3.23 & -0.84 \\
1984 & 3.8 & 0.13 & 1.59 & 2.10 \\
1985 & 2.7 & 0.19 & 1.69 & 0.86 \\
1986 & -3.4 & -0.14 & -1.89 & -1.33 \\
1987 & 1.9 & 0.07 & 1.04 & 0.84 \\
1988 & 1.5 & -0.19 & 0.85 & 0.80 \\
1989 & 4.4 & -0.01 & 2.05 & 2.40 \\
1990 & 5.2 & 0.25 & 2.28 & 2.67 \\
1991 & 4.2 & 0.11 & 1.16 & 2.91 \\
1992 & 3.8 & -0.04 & 1.50 & 2.32 \\
1993 & 1.8 & 0.13 & 0.10 & 1.55 \\
1994 & 4.5 & 0.04 & 1.63 & 2.88 \\
1995 & -6.5 & 0.04 & -2.66 & -3.85 \\
1996 & 5.6 & 0.16 & 3.39 & 2.06 \\
1997 & 7.1 & 0.01 & 3.23 & 3.88 \\
& & & & $($ Continúa) \\
& & & &
\end{tabular}


Moritz Cruz y Mayrén Polanco

(Continuación cuadro 3)

\begin{tabular}{lcccc}
\hline Año & Total & Agricultura & Industria & Servicios \\
\hline 1998 & 5.1 & 0.03 & 2.25 & 2.80 \\
1999 & 4.0 & 0.14 & 1.69 & 2.13 \\
2000 & 6.6 & 0.02 & 2.21 & 4.40 \\
2001 & -0.5 & 0.13 & -1.26 & 0.59 \\
2002 & 0.8 & 0.00 & -0.03 & 0.78 \\
2003 & 1.2 & 0.12 & -0.06 & 1.12 \\
2004 & 4.0 & 0.11 & 1.28 & 2.66 \\
2005 & 3.2 & -0.03 & 0.88 & 2.34 \\
2006 & 5.2 & 0.14 & 2.05 & 2.97 \\
2007 & 3.2 & 0.09 & 0.70 & 2.45 \\
2008 & 1.2 & 0.04 & -0.05 & 1.17 \\
2009 & -6.0 & -0.12 & -2.56 & -3.31 \\
2010 & 5.3 & 0.11 & 2.00 & 3.19 \\
2011 & 3.9 & -0.09 & 1.31 & 2.69 \\
2012 & 3.9 & 0.23 & 1.18 & 2.51 \\
\hline
\end{tabular}

Fuente: Elaboración propia con base en datos de WDI (en línea).

\section{La contribución del sector primario al estancamiento económico, 1970-2011: un ejercicio econométrico}

Desde una perspectiva kaldoriana, el determinante del crecimiento económico es el sector industrial, en particular la industria manufacturera (Kaldor, 1966). La idea está sintetizada en su primera ley del crecimiento, la cual sugiere que la dinámica del crecimiento del sector manufacturero determina la tasa de crecimiento del conjunto de la economía. ${ }^{10}$ Siguiendo esta idea, es decir, asumiendo que el sector industrial es el motor del crecimiento económico, un primer paso para conocer el papel del sector primario en el estancamiento económico consiste en realizar una estimación donde se incluyan las tasas de crecimiento de los sectores primario y terciario como variables explicativas del sector industrial. El objetivo es identificar si la dinámica del sector primario ha sido 
importante para la evolución del sector industrial. Para una economía exitosa, se esperaría que, tanto la dinámica del sector primario como la del sector servicios fuesen relevantes para el crecimiento del sector industrial. Esto debería reflejarse en parámetros con signos positivos (y estadísticamente significativos).

Para realizar éste, y los subsecuentes ejercicios econométricos, se utilizan datos anuales de la base de datos de las Naciones Unidas (disponible en: unstats.un.org) de 1970 a 2012. Las variables utilizadas son el valor agregado de cada sector productivo en millones de pesos reales $(2005=100)$. La razón para realizar el estudio a partir de 1970 radica, como se mencionó, en que el sector primario comenzó a ralentizar su dinámica expansiva hacia mediados de la década de 1960 (Rodríguez, 1980; Calva, 2012, y Moreno-Brid y Ros, 2009). En este sentido, se intenta identificar si el sector primario ha sido relevante para el industrial desde que inició la debacle del primero.

Como es estándar en el tipo de ejercicios que aquí se realiza, antes de proceder con la estimación debe conocerse el orden de integración de cada variable con el objeto de evitar resultados espurios. Para tal efecto se recurrió a los pruebas de Dickey-Fuller aumentada (ADF) y de Phillips-Perron (PP). La información contenida en el cuadro 4, de acuerdo a los resultados de dichas pruebas, indica que todas las variables (el valor agregado del sector primario [Pr], del sector industrial [In] y del sector servicios [Se]) tienen el mismo orden de integración, en este caso I (1), por lo cual no hay riesgo de estimar regresiones cuyos resultados arrojen asociaciones espurias.

Cuadro 4. Prueba de raíz unitaria

\begin{tabular}{lcc}
\hline Variable & ADF Estadístico ${ }^{t}$ & PP Estadístico $t$ \\
\hline LPr & -1.33 & -1.42 \\
$\mathrm{D}(\mathrm{LPr})$ & -8.01 & -8.04 \\
$\mathrm{Lln}$ & -2.42 & -2.78 \\
$\mathrm{D}(\mathrm{Lln})$ & -5.18 & -5.19 \\
$\mathrm{LSe}$ & -2.33 & -2.33 \\
$\mathrm{D}(\mathrm{LSe})$ & -4.62 & -4.62 \\
\hline
\end{tabular}

Notas: Pr: sector primario; In: sector industrial, y Se: sector servicios. L indica que la variable en cuestión está en logaritmos y D indica que la variable en cuestión ha sido diferenciada una vez. Las pruebas incluyen constante y el estadístico t indica el rechazo de la hipótesis nula de la existencia de raíz unitaria a 5\% de confianza.

Fuente: Elaboración propia usando E-views. 
Con este antecedente, estamos en condiciones de estimar una regresión donde la tasa de crecimiento del sector industrial (gIn) esté explicada por las tasas de crecimiento del sector primario $(\mathrm{gPr})$ y del sector terciario ( $\mathrm{gSe}$ ). Los resultados de tal estimación se muestran en el cuadro 5.

Cuadro 5. Determinantes del sector industrial, 1970-2012

MCO; variable dependiente: tasa de crecimiento del sector industrial $(\mathrm{g} \mid \mathrm{n})$

Variable explicativa

\begin{tabular}{lr}
\hline Constante & -1.82 \\
gPr & $(-3.3)$ \\
& -0.04 \\
gSe & $(-0.37)$ \\
& 1.49 \\
R2 & $(12.5)$ \\
& 0.80 \\
\hline Pruebas de diagnóstico & \\
\hline J-B normalidad & Valor p \\
B-G correlación serial (dos rezagos) & 0.1913 \\
ARCH & 0.6698 \\
WHITE & 0.4682 \\
RESET Ramsey & 0.4610 \\
\hline
\end{tabular}

Notas: estadísticos White † aparecen en paréntesis.

Fuente: Elaboración propia usando E-views.

Como puede observarse, la regresión (estimada por MCo) no presenta problemas asociados con normalidad, correlación serial, heterocedasticidad o forma funcional. Por otra parte, los coeficientes estimados son reveladores ya que indican, por un lado, que únicamente el sector servicios ha incidido en la dinámica del sector industrial durante los últimos 40 años; estrictamente hablando, una expansión de $1 \%$ de dicho sector genera un crecimiento de 
$1.5 \%$ en el sector industrial. Este resultado no es sustancialmente revelador si consideramos el crecimiento que ha observado el sector servicios durante el periodo de estudio, mismo que se ha reflejado en una creciente participación en el piB total.

Por otra parte, el coeficiente del sector primario tiene signo negativo, indicando que no contribuye positivamente al crecimiento económico. No obstante, este parámetro no es estadísticamente significativo, razón por la cual puede derivarse que la dinámica del sector primario no es (ha dejado de ser) relevante para la dinámica del sector industrial. Esto es un primer indicativo, como esperábamos, de que el sector primario ha dejado de contribuir, vía el sector industrial, al desarrollo económico.

Ahora bien, dado que todas las variables tienen el mismo orden de integración, es posible realizar un ejercicio que permita verificar si, pese a los resultados anteriores, hay una relación de largo plazo, siguiendo la idea kaldoriana antes expuesta. Para tal efecto, usando la metodología de cointegración de Johansen (Charemza y Deadman, 1997; Asteriou y Hall, 2011) y siguiendo el trabajo de Yao (2000), se estima primero un vector autoregresivo (VAR), incluyendo las mismas variables señaladas previamente, aunque esta vez en niveles transformadas por logaritmos. Recordemos que el procedimiento de Johansen es un método de máxima verosimilitud que utiliza sistemas de ecuaciones dinámicas, específicamente un modelo de autorregresión vectorial. Asimismo, esta metodología permite identificar no sólo la presencia de cointegración sino, de confirmarse, la cantidad de vectores cointegrados así como su especificación (Oreiro et al. 2012).

Es importante destacar que siguiendo la idea kaldoriana de que el motor de crecimiento es el sector industrial, se incluyen las variables en el var en el siguiente orden: primero, el sector industrial, después, el sector primario y, finalmente, el sector terciario. Del mismo modo, se decidió incluir dos variables dummy para capturar los efectos de periodos atípicos en la tendencia del crecimiento, es decir, a la crisis de 1995 y la recesión global de 2009. Ahora, con base en los criterios de información de Schwarz y de Akaike se estimó un $\operatorname{VAR}(1)$ entre las variables señaladas. En seguida, se realiza la prueba de cointegración de Johansen para identificar si hay algún vector de cointegración. De acuerdo al principio de Pantula (Asteriou y Hall, 2011) y a la prueba de la traza y del valor característico máximo del procedimiento de cointegración de Johansen es posible afirmar que las variables destacadas cointegran y que hay al menos un vector de cointegración (véase el cuadro 6). 
Moritz Cruz y Mayrén Polanco

Cuadro 6. Pruebas de cointegración

Prueba de la traza

\begin{tabular}{lccc}
\hline $\begin{array}{l}\text { Hipótesis } \\
\text { Núm. de corrección de errores }\end{array}$ & Valor propio & Estadístico de la traza & $\begin{array}{c}0.05 \\
\text { Valor crítico }\end{array}$ \\
\hline Ninguno* $^{\star}$ & 0.560137 & 58.51 & 35.19 \\
Al menos uno* & 0.336610 & 24.84 & 20.26 \\
Al menos dos & 0.177558 & 8.01 & 9.16 \\
\hline
\end{tabular}

Prueba del valor característico máximo

\begin{tabular}{lccc}
\hline $\begin{array}{l}\text { Hipótesis } \\
\text { Núm. de corrección de errores }\end{array}$ & Valor propio & $\begin{array}{c}\text { Máximo valor } \\
\text { característico }\end{array}$ & $\begin{array}{c}0.05 \\
\text { Valor crítico }\end{array}$ \\
\hline Ninguno* $^{*}$ & 0.560137 & 33.67 & 22.29 \\
Al menos uno* & 0.336610 & 16.82 & 15.89 \\
\hline
\end{tabular}

Notas: * rechazo de la hipótesis al nivel de confianza del 0.05; Mackinnon-Haug-Michelis (1999) p-values.

Fuente: Elaboración propia usando E-views.

Dado lo anterior, es posible estimar el vector de corrección de errores (VEC). La siguiente ecuación captura las elasticidades de largo plazo obtenidas del vector de cointegración (entre paréntesis, se muestra el estadístico $t$ ), es decir, el vector de cointegración normalizado es:

$$
\begin{aligned}
\operatorname{LIn}= & 23.34-2.52 \mathrm{LPr}+2.46 \mathrm{LSe} \\
& (3.25) \quad(-3.37) \quad(3.37)
\end{aligned}
$$

Los resultados de largo plazo, como puede observarse, confirman los previamente obtenidos. Esta vez, todas las variables son estadísticamente significativas. Así, por un lado, los sectores terciario e industrial mantienen una relación positiva y, derivado de nuestra especificación, es posible sugerir que el sector terciario coadyuva positivamente al crecimiento del sector industrial. Por el otro, el sector primario mantiene una relación inversa con el sector industrial y, por lo tanto, con el crecimiento económico, en este sentido, su evolución en vez de aportar al crecimiento, lo frena. La razón principal de este resultado puede deberse a la insuficiente inversión de que ha sido objeto 
el sector, resultado, como se ha señalado, del desmantelamiento de la política agraria, y, consecuentemente, de su crecimiento insuficiente.

Finalmente, el cuadro 7 presenta los parámetros de ajuste asociados al modelo vec. Su significancia estadística indica la endogeneidad o (débil) exogeneidad de cada variable. Como puede verse, todas las variables son endógenas, indicando que ninguna causa la evolución de las otras. Este resultado, por un lado, soporta el hallazgo de que el sector primario no promueve el crecimiento económico. Sin embargo, es revelador en el sentido de que ni el sector industrial ni el terciario se determinan mutuamente (ni al sector primario). Una posible explicación de esto podría estar dada por la debilidad de encadenamientos entre los sectores productivos.

Cuadro 7. Resultados del VEC

\begin{tabular}{lrrr}
\hline & $D(L \ln )$ & \multicolumn{1}{c}{$D(L P r)$} & \multicolumn{1}{c}{$D(L S e)$} \\
\hline Término de corrección de errores & -0.271645 & -0.189569 & -0.207023 \\
† estadístico & {$[-3.37]$} & {$[-4.33]$} & {$[-4.92060]$} \\
R2 ajustada & 0.282638 & 0.351951 & 0.472856 \\
\hline Estadístico LM (autocorrelación) & 0.3700 & & \\
White (heterocedasticidad) & 0.6427 & & \\
Prueba de Urzúa (normalidad) & 0.8149 & & \\
\hline
\end{tabular}

Fuente: Elaboración propia.

\section{CONCLUSIONES}

En este trabajo, usando evidencia descriptiva y econométrica, se explora la contribución del sector primario en el estancamiento económico mexicano durante el periodo de 1970 a 2012. Los resultados basados en el análisis descriptivo indican que el sector primario ha contribuido tanto al estancamiento económico como al reforzamiento de las restricciones externa e interna al crecimiento. Por otra parte, los resultados de la regresión indican que el sector primario no ha sido significativo para la dinámica del sector industrial durante el periodo de estudio; los resultados del ejercicio de cointegración sugieren, no obstante, que en el largo plazo ambos sectores sí mantienen una relación, aunque de carácter negativo, lo cual refuerza los hallazgos previos en el sentido de que el sector primario frena el crecimiento económico. En particular, las estimaciones aquí 
expuestas indican que en el largo plazo por cada punto porcentual que se expande el sector primario, el sector industrial se frena poco más de 2 por ciento.

Las implicaciones de política de los hallazgos son relevantes pues es claro que el éxito de la economía mexicana, por las razones expuestas a lo largo del trabajo, sólo será alcanzable si se logra que el sector primario contribuya nuevamente al crecimiento económico de manera positiva. Para lograr esto, es indispensable cambiar el sesgo anti-rural del actual modelo económico, lo cual implica poner en marcha una política agrícola que garantice la generación de un excedente productivo del sector primario, mismo que descansa en el crecimiento de su productividad. Esto implica necesariamente el apoyo del gobierno al sector, introduciendo o retomando políticas que garanticen insumos mejorados por medio del apoyo a la investigación, a la educación, a la aplicación de nuevo conocimiento y de técnicas modernas; también es necesario el apoyo financiero que facilite el acceso al crédito en condiciones preferentes y a los seguros; también es indispensable el desarrollo de infraestructura (para riego y almacenaje, por ejemplo), y el apoyo al transporte, a la comercialización y al procesamiento, así como el uso de garantías de precios para mantener la estabilidad de los ingresos de los productores; finalmente, la protección comercial podría coadyuvar de manera significativa al fortalecimiento del sector (Calva, 2007; Chang, 2009). No debería haber razón para que este tipo de política agraria que ha estado (y está) vigente en prácticamente todos los países exitosos sea retomada en México. Sin ella, se insiste, alcanzar un crecimiento económico rápido y sostenido será imposible.

\section{AGRADECIMIENTOS}

Los autores agradecen a Mildred Espíndola su apoyo en la construcción de las diferentes bases de datos. Asimismo, reconocen el apoyo financiero del proyecto UNAM-PAPIIT RN301213 para la realización de esta investigación.

\section{NOTAS}

1 No obstante, uno de los aspectos sobre los que sí se discute es la posibilidad de alcanzar el éxito económico basado exclusivamente en el sector primario (véase, por ejemplo, Adelman, 1984). En este contexto, hay evidencia empírica para un conjunto de economías en desarrollo que demuestra que el sector primario ha sido (y es) el motor del crecimiento económico (Tiffin e Irz, 2006). 
2 Al respecto, Yao (2000: 33-34) señala que “debido a una ambición irreal y pobre visión del futuro, la mayoría de los gobernantes de economías en desarrollo han tratado de industrializar sus economías a una velocidad tan rápida que el crecimiento del sector agrícola ha sido sofocado, resultando en [...] pobre desempeño de toda la economía”.

3 Es interesante destacar que el estancamiento de la economía mexicana ha ocurrido a pesar del éxito del modelo liderado por las exportaciones en su esencia; es decir, las exportaciones manufactureras han pasado a ser prácticamente el total de las exportaciones (poco más de 80\%) y su dinámica ha sido una de las más importantes en escala mundial, creciendo a una tasa promedio de 15\% durante el periodo 1981-2012. Esta paradoja ha generado un ingente interés por conocer las causas del lento crecimiento de la economía mexicana.

4 Es importante señalar que el sector primario contribuye también al crecimiento económico generando, tanto equilibrio regional como ecológico, además de mayor cohesión social (véanse, Haung y Bouis, 2001).

5 Es relevante apuntar que desde una perspectiva marxista también se sostiene la transferencia del valor del sector primario al resto del sector productivo (Valenzuela e Issac, 1999).

6 No es casual, en este sentido, observar desde esas fechas una marcada disminución de la participación del sector primario en el producto total.

7 Entre estas causas puede distinguirse, por un lado, la forzosa adopción de los programas de ajuste y estabilización del FMI y del BM, los cuales implicaron masivas reducciones del gasto público y, por otro, los múltiples problemas de eficiencia que aquejaban al sector como consecuencia del largo periodo en que permaneció altamente subsidiado y protegido (GómezOliver, 1996).

8 Calva (2012: 69) apunta, por ejemplo, que desde mediados de la década de los sesenta los precios de garantía dejaron de utilizarse como instrumento de fomento productivo para ser usados como ancla antiinflacionaria; dicho mecanismo fue, no obstante, reinstaurado temporalmente en la década de los setenta.

9 Dicho aumento fue además coyuntural, resultado del masivo influjo de capitales que la economía recibió en el periodo 1989-1994. Dichos flujos fueron esterilizados por el banco central y, como es sabido, tales operaciones implican un aumento del crédito interno.

10 Véase, entre otros, Felipe (1998), Dasgupta y Singh (2006), McCausland y Theodossiou, (2012) para evidencia empírica confirmando la primera ley del crecimiento de Kaldor.

\section{BIBLIOGRAFÍA}

Adelman, I. (1984), "Beyond export-led growth", World Development, vol. 12, núm. 9, pp. 937-949.

Asteriou, D., y S. Hall (2011), Applied econometrics, 2da. ed., Palgrave-Macmillam, Reino Unido. 
Bezemer, D., y D. Headey (2008), "Agriculture, development and urban bias", World Development, vol. 36, núm. 8, pp. 1342-1364.

Calva, J. L. (2012), "Políticas agropecuarias para la soberanía alimentaria y el desarrollo sostenido con equidad", en J. L. Calva (ed.), Politicas agropecuarias, forestales y pesqueras. Análisis estratégico para el desarrollo, vol. 9, Juan Pablos, pp. 67- 92.

(2007), "Políticas de desarrollo agropecuario", en J. Calva (ed.), Desarrollo agropecuario, forestal y pesquero. Agenda para el desarrollo, vol. 9, Miguel Ángel Porrúa, México, pp. 17-33.

(1988), Crisis agrícola y alimentaria en México 1982-1988, Fontamara, México.

Cruz, M.; A. Sánchez, y E. Amann (2011), "Mexico: food prices increases and growth constraints", CEPAL Review, núm. 105, pp. 73-86.

Chang, H-J. (2009), "Rethinking public policy in agriculture: lessons from history, distant and recent", Journal of Peasant Studies, vol. 36, núm. 3, pp. 477-515.

Charemza, W., y D. Deadman (1997), New directions in econometric practice, 2da. ed., Edward Elgar, Inglaterra.

Dasgupta, S., y A. Singh (2006), "Manufacturing, services and premature deindustrialisation in developing economies. A Kaldorian analysis", UNUWIDER research paper, núm 46, pp. 1-20.

Eckstein, S. (1968), El marco macroeconómico del problema agrario mexicano, Centro de Investigaciones Agrarias, México.

Felipe, J. (1998), "The role of the manufacturing sector in Southeast Asian development: a test of Kaldor's first law", Journal of Post Keynesian Economics, vol. 20, núm. 3, pp. 463-485.

Gollin, D.; S. Parente, y R. Rogerson (2002), "The role of agriculture in development”, The American Economic Review, vol. 92, núm. 2, pp. 160-164.

Gómez-Oliver, L. (1996), "El papel de la agricultura en el desarrollo de México", Estudios Agrarios, núm. 3, pp. 1-52

Haung, J., y H. Bouis (2001), "Structural changes in the demand for food in Asia: Empirical devidence from Taiwan”, Agricultural Economics, vol. 26, pp. 57-69

Henley, D. (2012), "The agrarian roots of industrial growth: rural development in South-East Asia and sub-Saharan Africa", Development Policy Review, vol. 30, núm. 51, pp. s25-s47.

Kaldor, N. (1966), Strategic factros in economic development, Ithaca, Nueva York. 
(1989), "Causes of the slow rate of economic growth in the United Kindom”, en F. Targetti, y A. Thirlwall (eds.), The essential Kaldor, Duckworth, Inglaterra, pp. 282-310.

Lewis, A. (1954), "Economic development with unlimited supplies of labour", The Manchester School, vol. 22, pp. 139-191.

McCausland, W., y I. Theodossiou (2012), "Is manufacturing still the engine of growth?", Journal of Post Keynesian Economics, vol. 35, pp. 79-92.

McCombie, J. S. L., y A. P. Thirlwall (2004), Essays on balance of payments constrained economic growth, Routledge, Londres.

Moreno-Brid, J. y J. Ros (2009), Development and growth of the mexican economy, Oxford University Press, Nueva York.

Oreiro J.; L. Nakabashi; G. Costa da Silva, y G. Guimarães e Souza (2012), "La economía del crecimiento impulsado por la demanda. Teoría y evidencia respecto del Brasil", Revista de la CEPAL, núm. 106, pp. 161-180.

Rodríguez, G. (1980), "Tendencias de la producción agropecuaria en las dos últimas décadas", Economía Mexicana, núm. 2, pp. 57-103.

Thirlwall, A. P. (2003), Growth and development, $7^{\mathrm{a}}$ ed. Palgrave-Macmillan, Nueva York.

Tiffin, R., y X. Irz (2006), "Is agriculture the engine of growth?", Agricultural Economics, vol. 35, pp. 79-89.

Yao, S. (2000), "How important is aigriculture in China’s economic growth?" Oxford Development Studies, vol. 28, núm. 1, pp. 33-49.

Valenzuela, J., y J. Isaac (1999), Explotación y despilfarro: análisis critico de la economía mexicana, Plaza y Valdés, México. 
